\title{
Cyclic voltammogram analysis of the environmental aspects of the use of ferrocenyl carbinols
}

\author{
Ahmad Khalaf Alkhawaldeh ${ }^{1}$ \\ Department of Pharmaceutical Chemistry; College of Pharmacy, Jerash University, \\ Jordan.
}

\begin{abstract}
Ferrocene and its derivatives have ecologically effective antidetane properties. In this regard, ferrocene reacts with cyclic ketones and ferrosenylcarbinols are synthesized. It should be noted that ferrocene enters into electrophilic reactions and the process takes place in an acidic environment. In addition, the yield of the new product was small compared to the reactions of ferrocene with non-cyclic ketones. This is due to the spatial structures of molecules. The elemental analysis of obtained compounds was carried out; the structures were researched by cyclic voltammograms and Chronoamperometric.
\end{abstract}

Keywords: Cyclic voltammograms, Chronoamperometric, Cyclic ketones structure; Environment ecological aspects ferrocene; Ecological aspects

\section{Introduction}

In the literature, it is known that ferrocene can reduce smoke in combustible hydrocarbons, while cymantrene is more efficacious than antidetonants. Recently, due to complications of ecological problems mostly caused by cars, there has been a clear shift in research, caused by the development of technology and increased demand for ecologically pure combustion fuels and their products (Alkhawaldeh, 2020).

In this context, new organometallic (aliphatic, cyclic and heterocyclic) compounds are being searched and used with a variety of functional groups of most transition metals. Iron is necessary for the ecosystem and is non-toxic. Iron, as we all know, plays a critical role in the ecosystem. This element is also very important in the biosphere. Iron is required for photosynthesis (cytochromes, ferrodoxins), as well as a variety of enzymes. In the absence of iron, the formation of chlorophyll is delayed. Chlorosis (yellowing) and loss of leaf color are possible, especially when young. With prolonged iron deficiency, dense grasses die off at the edges of the leaf blade, tree shoots die off, overall productivity decreases, and plant resistance to diseases decreases( Alkhawaldeh, 2021).

In addition, it should be noted that iron compounds are also effective in the petrochemical industry. Many substances that replace the environmentally hazardous tetraethyl lead as a fuel additive have been tested in motor vehicles. Although much work has been done to make fuel additives more effective in this direction, more effective methods are still needed and work is underway (Alkhawaldeh, 2020).

From this point of view, various additives are added to fuels, and for this purpose, we have synthesized ferrocenyl-type metal organic complexes added as additives to fuels. Ferrium dicyclopentadienes and their derivatives possess many important properties. That is why these compounds are being used in pharmacy, petroleum chemistry, in preparation of anti-corrosion materials. Compounds derived from ferrocene-alkyl, aryl derivatives, carbonyls, ethers and esters are of special interest. Such highly soluble organometallic compounds are efficient neutralizers of unbranned residue, due to their high dispersal, in the relevant hydrocarbon fuel.

It is known that reaction of alyphatic ketones with ferrocene is easier than that of cyclic ketones, and yield is higher. It is worth noting that there are many questions concerning production of ferrocenylcyclokarbonyls. Reactions between alyphatic ketones and ferrocene in the presence of catalyst $\mathrm{H}_{2} \mathrm{SO}_{4} / \mathrm{DEAN}$ (diethylammonium naphthenate) (2:1) have been conducted and corresponding ferrocenylcarbonyls have been synthesized. As a follow-up to research reaction between cyclic ketones and ferrocene has been analyzed (Alkhawaldeh, 2020). 


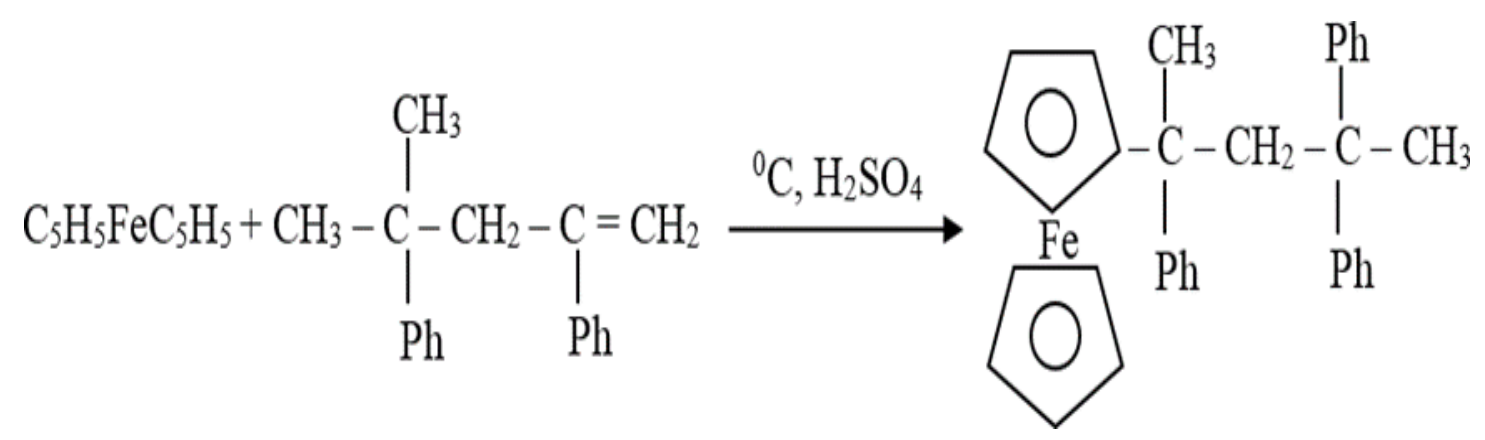

Scheme 1 follows:

Since these reactions take place in an acidic environment, the mechanism of the process can be described as

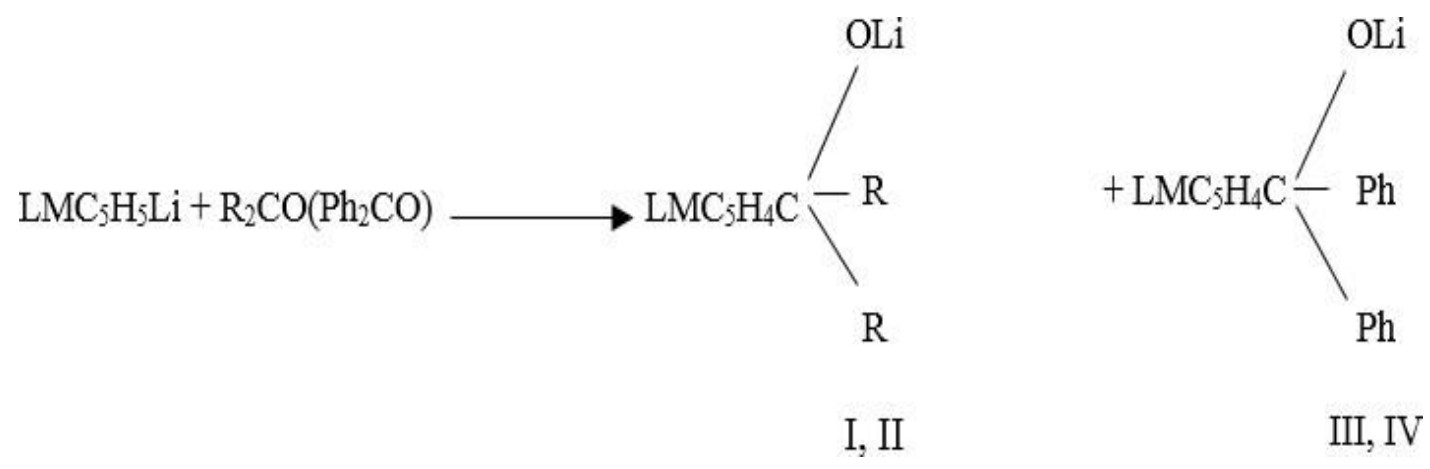

Scheme 2

It is known that reaction of aliphatic ketones with ferrocene is easier than that of cyclic ketones, and yield is higher. It is worth noting that there are many questions concerning production of ferrocenylcyclokarbonyls. For this purposes, it has been tested to use derivatives of ferrocene by substituting metals, for example, $\mathrm{C} 5 \mathrm{H} 5 \mathrm{FeC} 5 \mathrm{H} 4 \mathrm{Li}$ or $\mathrm{C}_{5} \mathrm{H}_{5} \mathrm{FeC} 5 \mathrm{H}_{4} \mathrm{MgHal}$ and corresponding cyclic ketones in the THF solvent. When comparing the reactions that are shown above, it had been observed that ferrocenylcyclopentanol (1) has better yielded than ferrocenylcyclohexanol (2). All of cyclic ketones bearing 4 carbon atoms (cyclobutanols) does not take part in these reactions. There is strain since the angle between $\mathrm{C}$-atoms are much smaller than normal tetrahedr, which is 109028'. Due to the Bayer strain in 5- and 6- membered cycles $\left(00,44^{\prime}\right)$ system is somewhat unstable. The starin in 6-membered cycles is about $(-50,16$ '). Best result has been obtained on cyclohexanone. Due to its stability thanks to existence of $\alpha$, $\beta$-conformations, there has been monosubstitution in nucleus of ferrocene and ferrocenylcyclohexanol (2) has been produced.Main conditions required for reaction are: acidic medium, and maintanience of temperature at required level.

Since the reaction is exothermic the temperature of medium increases by time and Van-der-Waals forces between ketones are being weakened. Due to deformation of molecule, structure the yield of reaction decreases. On the other hand, we must also take into account steric factors, which are the reason for low yield of aliphatic ketones. We would like to note that we have also carried out the similar reactions with aliphatic ketones and the yield was about 75 $78 \%$. The conditions, nature of catalyst, its concentration, temperature of system and other factors must be taken into account too.

The reaction of hydrogen sulfide and some mercaptanes with both aliphatic and aromatic has been analysed. This way ferrocenylthioethers which have bad smell has been obtained. These compounds are solved in nearly all of organic solvents, especially benzene. This property can be utilised in order to clean some oils containing mercaptane or its fractions. Considering that, currently hazardous substances from exhaust gases, especially unburnt residues, are the most pressing and urgent environmental problems for diesel and non-standard motor fuels that contaminate air. It is well known that replacement of aromatic core by ferrocene in organic compounds produces products with properties not characteristic of or less expressed in the initial compounds (Alkhawaldeh, 2020).

The unusual pharmacokinetics and biodegradation of ferrocene derivatives in the body are often associated with this. This approach is currently being actively exploited in the search for new drugs. Several compounds under clinical trial were developed, such as ferrocifen and ferroquine, as derivatives of well-known medications with established biologic activity (an analog of chloroquine). The modification of different, including natural, heterocyclic derivatives with ferrocene is a popular research area. Comparative analysis of ferrocenyl carbinols properties in ecological aspects was carried out. 


\section{Experimental}

Carbinol and carbonilate derivatives of ferrocene are synthesized. To achieve this, 0.1 mol of ferrocene (cymantrene) is first melallated with $25 \mathrm{ml}$ of $1,2 \mathrm{NH}-\mathrm{BuLi}$, and then processed with 0.1 mol of carbonyl compound at $-10^{\circ} \mathrm{C}$. Under phase-transfer catalysis, carbinol derivatives of ferrocene are synthesized. To achieve this, an immiscible diphase system (water/petroleum ether) is first created, with the lower layer being inorganic and the upper layer being organic. Prior to the syntheses, the solvents were purified using standard procedures. Chemical shifts are expressed in parts per million (ppm) in comparison to residual or deuterated solvent signals. A Bibby Scientific SMP30 capillary melting point apparatus was used to determine the melting points. TLC on Silufol UV 254 plates was used to check the purity of the compounds. The Bosch method was used to determine the analyte on motor D-20. The method entails passing a specific amount of analyte motor gases through filtering material and weighing the amount of furnace black in 11 gas in $\mathrm{mg}$.

\section{Results and Discussions}

The yield of goals of the reaction, (1) and (2) beiing lower means that thera is considerable amount of loss, and as we noted before, which can be explained by the deformation of molecules taking part in reaction, which leads to less productive collisions.

In the case described above it has been observed that reactive ability with cyclopentanone (1) is less than (2) which reflects upon reaction products. $92-94 \% \mathrm{H}_{2} \mathrm{SO}_{4}$ has been used as catalyst purposes. The goal of using concentrated acid is because of creating acidic medium and the oxidative property of it which speeds up the process. As we mentioned, reaction happens in acidic medium. Obtained ferrocenylcyclopentanol (1) and ferrocenylcyclohexanol (2) has been washed by distilled water few times and dried under the air for a day. Both products are orange powder with specific smell.

Brutto formula of compound (1) is $\mathrm{C}_{15} \mathrm{H}_{18} \mathrm{FeO}$, and that of (2) is $\mathrm{C}_{16} \mathrm{H}_{20} \mathrm{FeO}$. Elemental analysis of compounds (1) and (2) gives the following results:

\begin{tabular}{|l|l|l|l|}
\hline & $\mathrm{C} \%$ & $\mathrm{H} \%$ & $\mathrm{O} \%$ \\
\hline $\mathrm{C}_{15} \mathrm{H}_{18} \mathrm{FeO}$ & 75.66 & 6.61 & 7.92 \\
\hline $\mathrm{C}_{16} \mathrm{H}_{20} \mathrm{FeO}$ & 72.60 & 8.04 & 5.93 \\
\hline
\end{tabular}

On the PMR spectrum of unsubstituted ferrocene because of symmetric nature of cyclopentadiene (C5H5) ligands the protons had signals at $4.30 \mathrm{ppm}$. After the substitution the protons of unsubstituted cyclopentadiene was at $4.17 \mathrm{ppm}$, while that of substituted cyclopentadienes (1) and (2) were at $4.57 \mathrm{ppm}$ and $4.55 \mathrm{ppm}$ for the protons respectively. The PMR spectra was taken in CCl4 solution, and TMS was used as standard reference. 


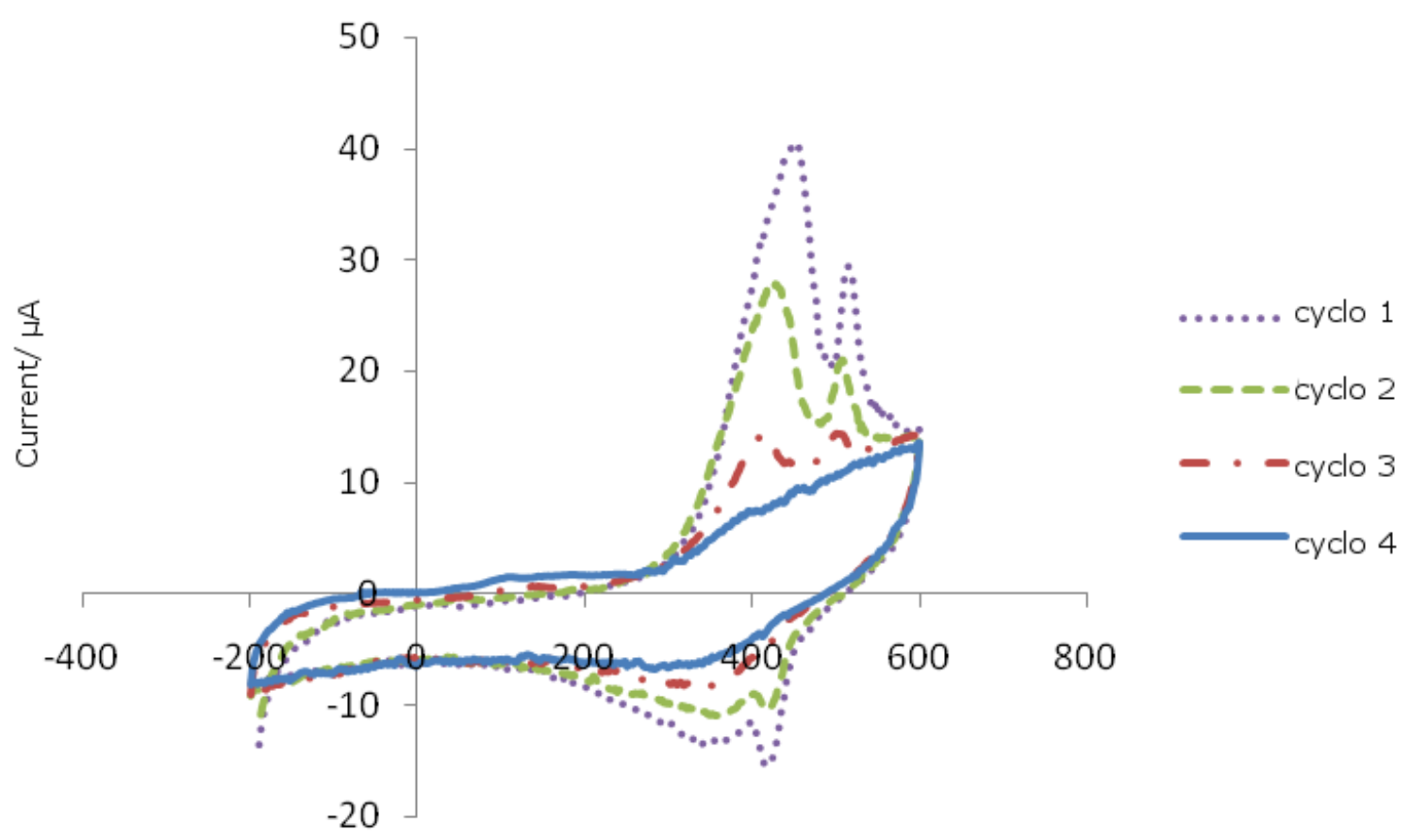

Figure 1. Cyclic voltammograms of ferrocenylcyclopentanol

The IR spectral analysis of ferrocenylcyclopentanol (1) and ferrocenylcyclohexanol (2) has been run. The peaks for OH residue was observed on $v \mathrm{OH} 3420-3466 \mathrm{~cm}^{-1}$ for (1) and $v \mathrm{OH} 3440-3468 \mathrm{~cm}^{-1}$ on (2). Note that since the spectra are very close to each other, the spectrum of only one of them is given below (1).The peaks for ketone (CO) residue has not been observed on the characteristic $\mathrm{vOH}\left(1680-1690 \mathrm{~cm}^{-1}\right)$ area. It shows that original ketone used as reagent has been consumed to get alcohol, whereas rest has undergone some transformations. When same reactions were carried out fot aliphatic ketones the signals for ketone (CO) residue was still absent. Moreover it has been observed that $\mathrm{OH}$ residue on ferrocenylcarbynol is of dual nature.

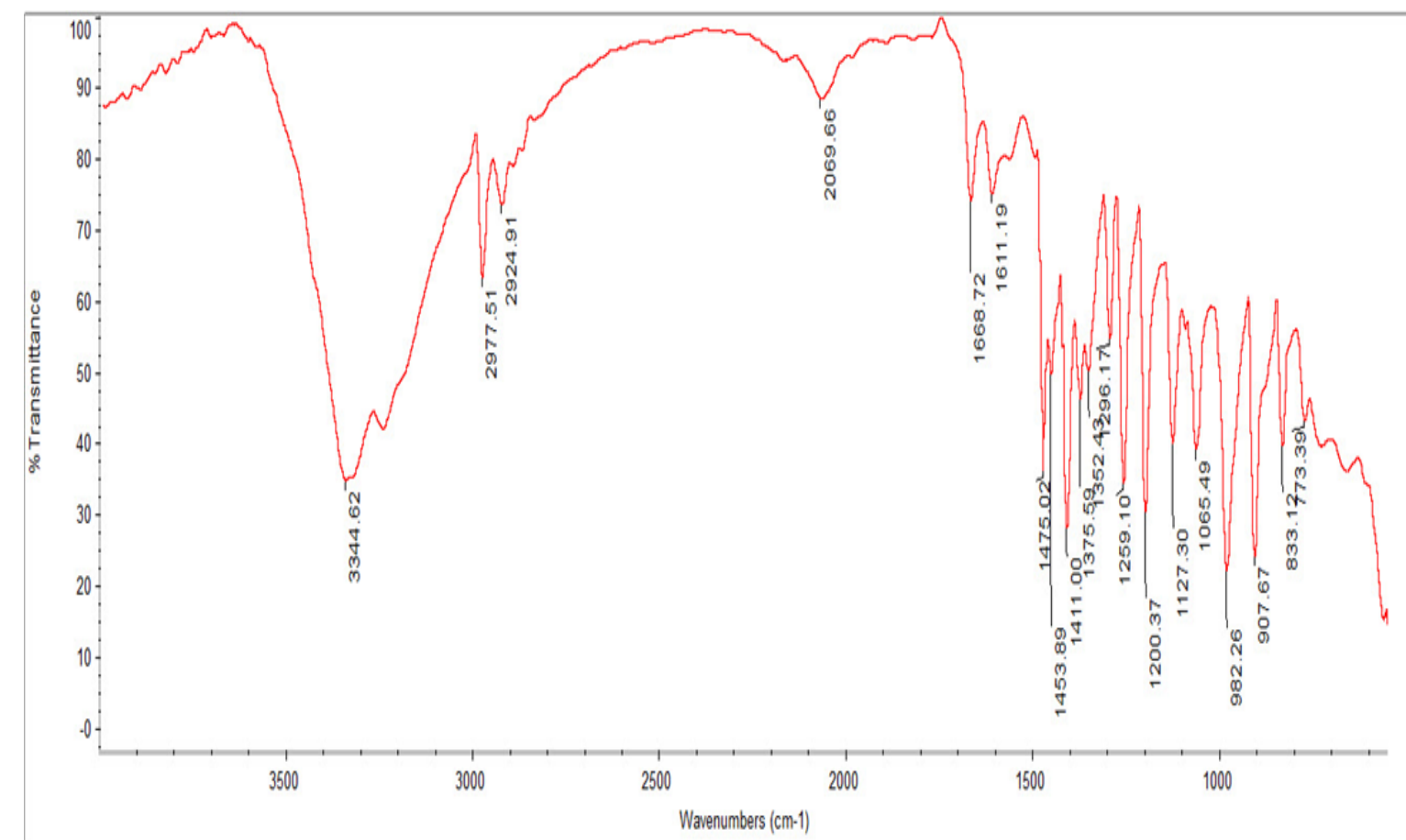

Figure 2. IR spectral analysis of ferrocenylcyclopentanol 
As a conclusion of study of PMR and IR spectra of ferrocenylcarbynols, it has been learned that there are two $\mathrm{OH}$ groups in these metalcomlexes. One of them was is in intramolecular coordination with central $\mathrm{Fe}$ atom, while another $\mathrm{OH}$ is free.

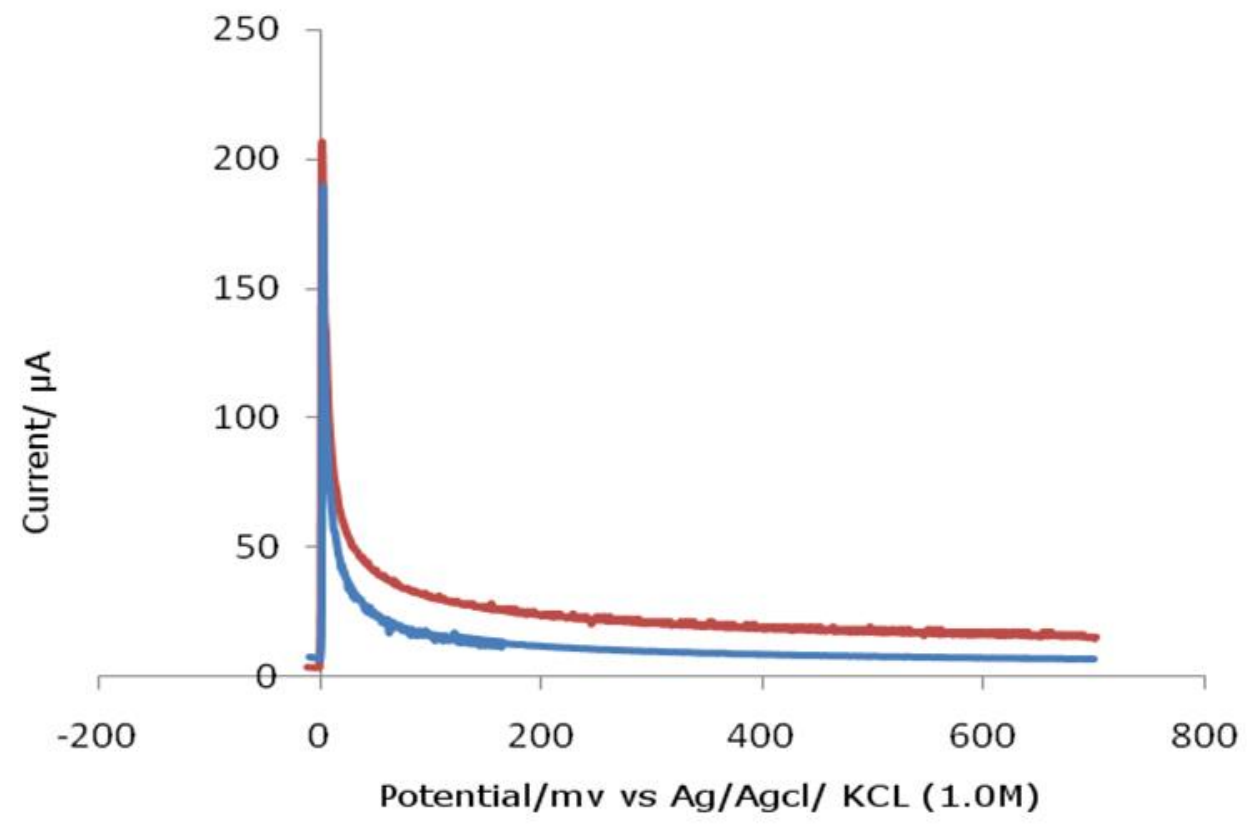

Figure 3. Chronoamperometric analysis of ferrocenylcyclopentanol

As we know, the flue gases emitted into the atmosphere contain sulfur compounds, which cause global warming. In this regard, minimizing the amount of sulfur in the fuel is very important from an environmental point of view. One of the most interesting reactions from the ecological point of view is the reaction of ferrosenylcarbinol metal complexes with the $\mathrm{H} 2 \mathrm{~S}$ reaction at a temperature of $200 \mathrm{C}$ in a THF environment. When hydrogen sulfide is taken in a 1: 1 ratio with a metal complex, mono-substituted derivatives of ferrocene are obtained. When taken in a ratio of 2: 1 , two-phase thio derivatives are obtained.

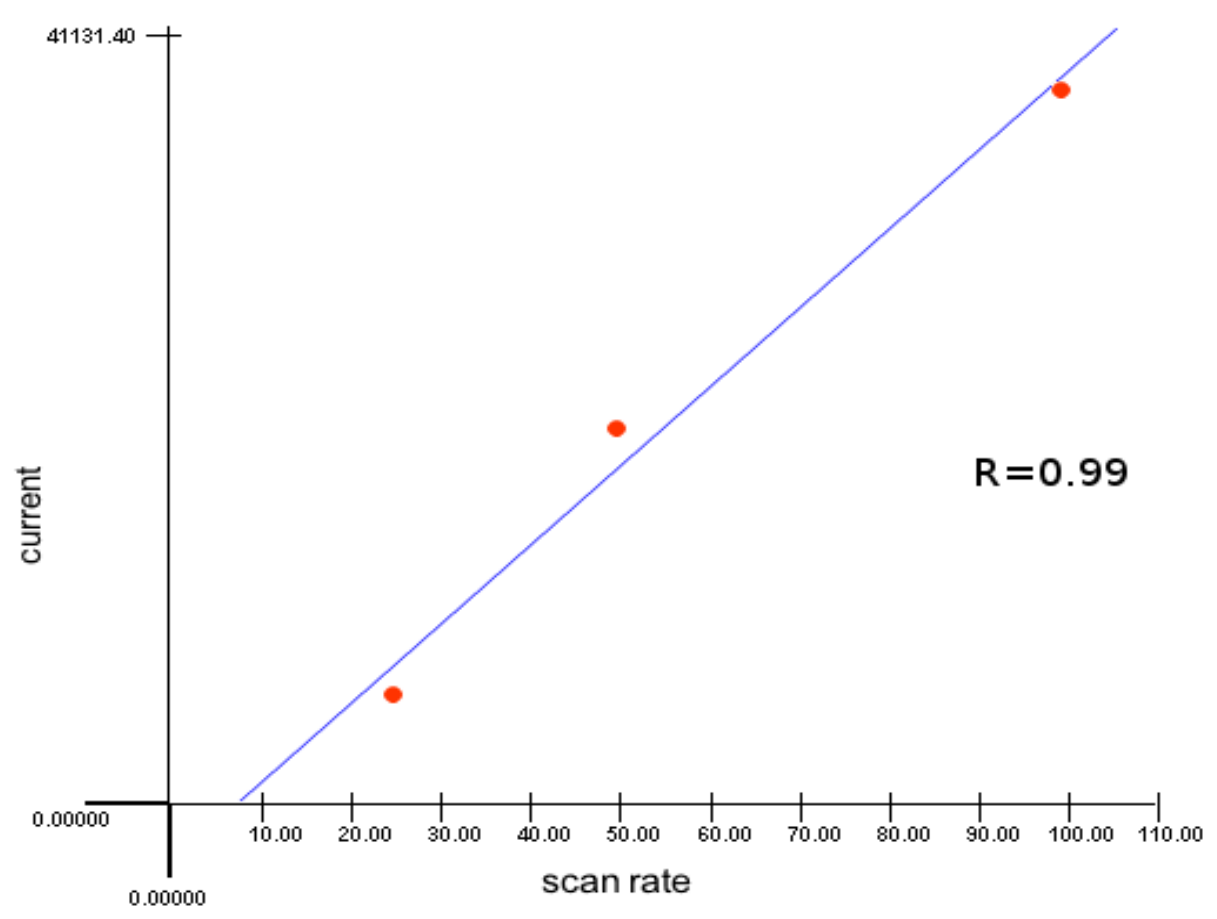

Figure 4. Current vs. time of ferrocenylcyclopentanol 
The reactions considered are that very important from an environmental point of view. It has been proven that such compounds can increase their octane number by 10-12 units, depending on the composition of gasoline. In this regard, the new ferrosenylcarbinols synthesized for the first time under interfacial catalysis are also of practical importance.

\section{Conclusions}

New ferrosenyl carbinols have been synthesized between ferrocene and cyclic ketones under conditions of interphase catalysis (H2SO4/DEAN). The ratio between sulfuric acid and DEAN was 1:2. The resulting ferrosenyl carbinols were obtained in lower yields than open chain ketones. The structure of the obtained ferrosenyl carbinols was determined by IR and PMR methods. Ferrosenyl carbinols are considered to be better fuel additives than ferrocene. The reactions of ferrocenyl carbinols with hydrogen sulfide are also considered. Obtained ferrosenylcarbinols are highly effective additives for fuels that are environmentally friendly substitutes for tetraethyl lead.

\section{References}

Alkhawaldeh, A. K., M.Krishan, M., Altwaiq, A., Dabaibeh, R. N. (2020). Preparation of Nanostructured/ Microplatinum Surfaces by Application of a Square Wave Potential Regime for Methanol Oxidation. Eurasian Journal of Analytical Chemistry, 15(1), emEJAC-00362.

Alkhawaldeh, A. K., (2020). Analytics of Antimony in Natural Water of Nanoparticle Platinum Electrode by Application Square Wave Voltammetry. International Journal of Multidisciplinary Sciences and Advanced Technology, 1(4): 96-103.

Alkhawaldeh, A. K., (2020). COVID-19: Simultaneous Surveillance Studies and Case Series, Jordan as a Case Study. International Journal of Multidisciplinary Sciences and Advanced Technology. Special Issue 1: Covid-19: 55-62.

Alkhawaldeh, A. K., (2020). Electrical Conductivity of Natural Volcanic Tuff Mix by Cyclic Voltammetry Method, International Journal of Multidisciplinary Sciences and Advanced Technology, 1(5): 37-44.

Alkhawaldeh, A. K., (2020). Platinum nanoparticle electrode modified iodine used cyclic voltammetry and chronoamperometric for determination of ascorbic acid. Analytical and Bioanalytical Electrochemistry, 12 (6): 780-792.

Alkhawaldeh, A. K., (2020). Platinum Nanoparticle in Tantalum Electrode for the Electrochemical Analysis of Heavy Metal Ions. International Journal of Intelligent Computing and Technology, 4 (1): 25-35.

Alkhawaldeh, A. K., (2020). Platinum Nanoparticles for the Electrochemical Study of Heavy Metal ions Formed by the Sputtering Deposition of the ion beam Electrode. International Journal of Engineering and Artificial Intelligence. 1(3): 1-8.

Alkhawaldeh, A. K., Alzawahreh, A., Alkhawaldeh, R., (2021). Electrochemical Sensors and Determination for Heavy Metal by Rotating Disk Platinum Electrode and Chronoamperometric Method. International Journal of Engineering and Artificial Intelligence, 2 (1): 17-26.

Alkhawaldeh, A. K., and Alkhawaldeh, R. (2020). Highly Sensitive copper Heavy Metal Analysis on Nanoparticle Platinum and palladium electrode, International Journal of Engineering and Artificial Intelligence. 1(2): 33-39.

Alkhawaldeh, A. K., (2020). Platinum Nanoparticles for the Electrochemical Study of Heavy Metal ions Formed by the Sputtering Deposition of the ion beam Electrode. International Journal of Engineering and Artificial Intelligence. 1(3): 1-8.

Alkhawaldeh, A. K., (2021). Electrochemical Analysis of Heavy metal by Cyclic Voltammetry Method. International Journal of Engineering and Artificial Intelligence, 2 (2): 27-33.

Alkhawaldeh, A. K., (2021). Platinum nanoparticle electrode electrochemical lead (II) determination with square wave voltammetry modified with iodine. AIP Conference Proceedings, 2339 (1): 020221 (2021); https://doi.org/10.1063/5.0045328.

Alkhawaldeh, A. K., Abdel Hadi Al Jafari (2021). Electrochemical Sensors and Determination for silver ion by Cyclic Voltammetry at iodine-coated Platinum nanoparticles electrode. Annals of the Romanian Society for Cell Biology, 25 (6): 20280 - 20291. 
Ahmad Khalaf Alkhawaldeh. (2021). Technology patterns in Nanochemistry Based on GII Indicator. International Journal of Engineering and Artificial Intelligence, 2 (3). (2021): 28-32.

Ahmad Khalaf Alkhawaldeh. (2021). Cyclic voltammogram analysis of the environmental aspects of the use of ferrocenyl carbinols. International Journal of Engineering and Artificial Intelligence, 2 (4). (2021): 7-12. 